\title{
Prognosis value of RBBP8 expression in plasma cell myeloma
}

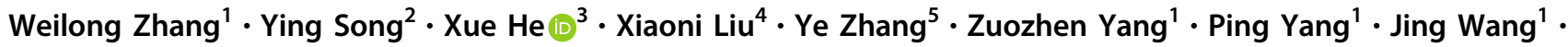 \\ Kai Hu${ }^{1} \cdot$ Weiyou $\mathrm{Liu}^{4} \cdot$ Xiuru Zhang ${ }^{3} \cdot$ Xiaoliang Yuan $^{4} \cdot$ Hongmei Jing ${ }^{1}$
}

Received: 30 July 2018 / Revised: 30 October 2018 / Accepted: 2 November 2018 / Published online: 9 January 2019

(c) The Author(s) 2019. This article is published with open access

\begin{abstract}
Plasma cell myeloma (PCM) secretes monoclonal immunoglobulin (Ig) by clonal plasma cells of abnormal proliferation in the bone marrow. As PCM is incurable, it is necessary to find new biomarkers to predict the prognosis and recurrence of PCM. The relationship between cancer and RBBP8 has not been fully studied. The role of RBBP8 in tumorigenesis remains inconsistent. We described the expression of RBBP8 in the gene expression profile of 1930 PCM samples (1878 PCM patients) from seven independent data sets. We analyzed the relationship between RBBP8 and survival prognosis, recurrence, and treatment response in patients with PCM, and the biological significance of RBBP8 in PCM. The gene expression level of RBBP8 was significantly related to the International staging system (ISS) grade of PCM $(P=0.0012)$. RBBP8 expression in different molecular subtypes was different $(P<2.2 \mathrm{e}-16)$. High RBBP8 expression is associated with poor survival in PCM $(P<0.0001)$. High expression of RBBP8 indicates that PCM patients are more likely to relapse $(P=$ 0.0078). The biological significance of RBBP8 in PCM is related to the cell cycle $(P<0.05)$. High RBBP8 expression predicts poorer survival and more likely relapse in PCM. RBBP8 plays an important role in the cell cycle of PCM. RBBP8 can be considered an independent prognostic factor for PCM. RBBP8 can be used as a potential biomarker for assessing the prognosis of PCM patients.
\end{abstract}

These authors contributed equally: Weilong Zhang, Ying Song, Xue $\mathrm{He}$

Supplementary information The online version of this article (https:// doi.org/10.1038/s41417-018-0069-3) contains supplementary material, which is available to authorized users.

Xiuru Zhang

zhangxiuru999@163.com

$\triangle$ Xiaoliang Yuan

yxlyyxs@126.com

$\triangle$ Hongmei Jing drjinghm@163.com

1 Department of Hematology, Lymphoma Research Center, Peking University Third Hospital, Beijing 100191, China

2 Gannan Medical University, Ganzhou 341000, China

3 Department of Pathology, Beijing Tiantan Hospital Affiliated with Capital Medical University, No. 6 Tiantan Xili, Beijing 100050, China

4 Department of Respiratory Medicine, The First Affiliated Hospital of Gannan Medical University, Ganzhou 341000, China

5 Melbourne School of Population and Global Health, The University of Melbourne, Victoria 3010, Australia

\section{Introduction}

Plasma cell myeloma (PCM) secretes monoclonal immunoglobulin (Ig) by clonal plasma cells of abnormal proliferation in the bone marrow. Clinical features of PCM typically present with bone damage, hypercalcemia, renal impairment, and anemia. PCM accounts for $\sim 10 \%$ of cancers in the blood system [1]. The average median survival of the disease is 3-4 years. Costa LJ summarized that the early mortality was $4-25 \%$ of newly diagnosed PCM patients in a randomized phase 3 clinical trial in the past decade [2]. However, he found that the early mortality rate is more higher than what has been reported in clinical trials [2]. At present, the treatment of novel myeloma drugs of immunomodulatory agents and proteasome inhibitors improve survival rates [3, 4]. In addition, combinations of high dose-chemotherapy with autologous stem cell transplantation (ASCT) resulted in better overall survival (OS) [5-7]. However, there is still a high recurrence rate in PCM. Therefore, it is necessary to find new biomarkers to predict the prognosis and recurrence of PCM.

According to the genetic classification, PCM can be classified based on the translocation and cyclin D (TC) 
and the University of Arkansas for Medical Sciences (UAMS) system. The TC classification distinguishes eight subgroups by the deactivation of primary immunoglobulin $\mathrm{H}$ translocations and transcriptional activation of cyclin D gene [8]. The UAMS molecular classification were classified into seven subtypes by different gene expression profiles, including MMSET $[\mathrm{t}(4 ; 14)]$, MAF $[\mathrm{t}(14 ; 16) / \mathrm{t}$ $(14 ; 20)], \mathrm{CD} 1 / 2$ [t(11;14), and t(6;14)], HY (hyper diploid cluster), PR (proliferation), and a cluster mainly characterized by a low percentage of bone disease (LB) [9] Based on the UAMS classification in 2010, PCM is reclassified as $\mathrm{CD} 1, \mathrm{CD} 2, \mathrm{CTA}, \mathrm{HY}, \mathrm{MF}, \mathrm{MS}$, myeloid, NFKB, and PR [10]. According to treatment response with bortezomib and dexamethasone (Dex), patients were divided into $\mathrm{CR}$ (complete response) group, PR (partial response) group, MR (minimum response) group, $\mathrm{NC}$ (no change) group, and PD (progressive disease) group [11]. Similarly, according to the treatment response with after induction chemotherapy (pre-1st) and after ASCT, patients were classified into CR group, VGPR (very good partial response) group, PR group, NR (stable disease) group, and Prog (no response, progressive disease) group [12].

The human RBBP8 (retinoblastoma-binding protein 8), also known as CTIP (CTBP (C-terminal-binding protein)interacting protein), is a protein coding gene. This protein interacts with other factors and participates in a variety of nuclear pathways. Overexpression of RBBP8 in tumors is mainly related to cyclin D1 transcription [13]. CTIP/RBBP8 gene accelerates tumorigenesis through transcriptional activity [14]. CTIP/RBBP8 was described as a key checkpoint of $\mathrm{G}_{1}$ phase by initiation S-phase and DNA replication [15]. CTIP/RBBP8 mediates DNA double-strand breaks repair in the cell cycle through homologous recombination [16, 17]. CTIP/RBBP8 interacts with proliferating cell nuclear antigen (PCNA) at specific localization and activates DNA damage checkpoints leading to DNA damage, suppressing DNA replication at $\mathrm{S}$ and G2 phases [18]. However, the relationship between cancer and RBBP8 has not been fully studied.

Investigation found RBBP8 genes was associated with sporadic brain arteriovenous malformations [19]. Advanced invasive bladder cancer was associated with the deletion of nuclear RBBP8 protein [20]. Deletion of the RBBP8 gene was associated with significantly worse prognosis in ovarian cancer [21]. The poor prognosis of breast cancer was related to the low or no expression of RBBP8 [22, 23]. RBBP8 is also overexpressed in certain tumors [24]. However, despite these associations with cancer, the expression level of RBBP8 has not been reported in PCM. We analyzed the association of RBBP8 expression with PCM prognosis, relapse, and event-free survival (EFS) or OS.

\section{Materials and methods}

\section{Data source and gene expression analysis}

Probe set measurements for all arrays were calculated using the RMA (robust multiarray averaging) method. Logarithmic conversion of relative RNA expression values was performed using $\log 2$. According to the RBBP8 gene expression level, RBBP8-high group, and RBBP8-low group using survminer package with maximally selected rank statistics arithmetic. Only genes with foldchanges $(\log 2)>0.8$ or $<-0.8$ were considered different expressed genes. $P$ values $<0.05$ were defined to be statistically significant. We obtained seven independent data set totally 1930 PCM samples (1878 PCM patients) from the seven independent Gene Expression Omnibus (GEO) data sets. This study was in accordance with the Helsinki Declaration.

GSE24080 of 559 patients were obtained from the GEO database. The gene expression was detected by Affymetrix Human Genome U133 Plus 2.0 Array [25]. We analyzed the relationship between RBBP8 expression and International staging system (ISS) stage, 1q21 amplification, molecular subtype, and survival.

GSE19784 of 311 patients were obtained from the GEO database. The gene expression was detected by using Affymetrix GeneChip U133 plus 2.0 arrays [10]. Hierarchical clustering identified 10 distinct subgroups. We analyzed the relationship between RBBP8 expression and molecular subtype (9 subgroups).

GSE9782 of 477 patients were obtained from the GEO database. Gene expression profiling was detected using the Affymetrix $133 \mathrm{~A} / \mathrm{B}$ microarray [11]. We analyzed RBBP8 expression in different therapeutic response with bortezomib or dexamethasone (Dex).

GSE83503 of 585 patients were obtained from the GEO database. The gene expression array was Affymetrix Human Exon 1.0 ST Array [26]. We analyzed the relationship between RBBP8 and PCM recurrence.

GSE82307 of 66 samples (33 patients) were obtained from the GEO database. Samples were tested by Affymetrix Human Genome U133 Plus 2.0 Array [27]. We analyzed the relationship between RBBP8 expression in presentation (baseline) and recurrence.

GSE19554 of 38 samples (19 patients) were obtained from the GEO database. Samples were tested by Affymetrix Human Genome U133 Plus 2.0 Array [28]. We analyzed the relationship between RBBP8 expression at diagnosis (baseline) and after induction chemotherapy (pre-1st).

GSE39754 of 136 patients were obtained from the GEO database. The gene expression was detected by Affymetrix Human Exon 1.0 ST Array [12]. We analyzed RBBP8 expression in different therapeutic response with the pre-1st and after ASCT. 
A type 审 2 审 3 安 4+

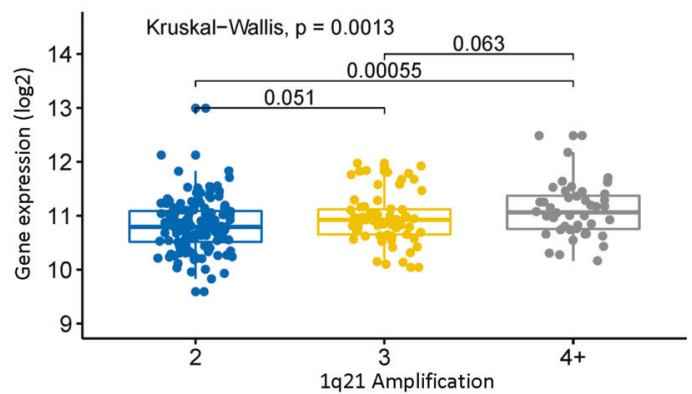

B

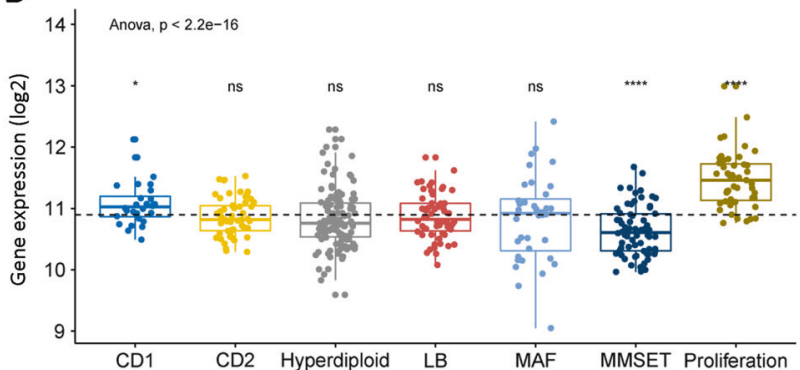

Fig. 1 RBBP8 gene expression in different molecular types of PCM. a Comparison of RBBP8 expression levels at different amplification levels of 1q21, The $X$ axis represents the 1q21 amplification level and the $Y$ axis represents gene expression. RBBP8 gene expression was measured as $\log 2 . P=0.0013$, Kruskal-Wallis test. $\mathbf{b}$ Comparison of RBBP8 expression levels in PCM of seven molecular subtypes. The $X$ axis represents different molecular subtypes and the $Y$ axis represents gene expression $(\log 2) . P<2.2 \mathrm{e}-16$, ANOVA test, ns, $*$ and $* * * *$ indicate $P>0.05, P \leq 0.05$, and $P \leq 0.0001$, respectively. The average of the entire data is used as a reference group. Each group level is compared with the reference group. Add a horizontal dashed line at the average of reference group

\section{Gene ontology (G0) analysis}

Pathway enrichment was performed using the DAVID tool with default parameters to analyze different expression genes between RBBP8-high groups and RBBP8-low groups in PCM (from data set GSE24080). GO pathway analysis results are showed in Fig. 4b.

\section{Statistics}

This study used R software v3.1.3 (ggplot2 and survminer package) for statistical analysis. Kruskal-Wallis test was used for comparison of RBBP8 expression levels between ISS grades. RBBP8 expression levels were compared between different molecular typing groups and different treatment response groups using one-way analysis of variance. Kaplan-Meier curves, log-rank test, Cox regression are used in survival analysis. RBBP8 expression comparison of 33 pairs specimens before and after recurrence and 19 pairs specimens before and after treatment using
Wilcoxon test. For all statistical methods, the $P<0.05$ was considered to indicate statistical significance.

\section{Results}

\section{Expression level of RBBP8 in different molecular subtypes of PCM}

The amplification of 1q21 was related to the expression level of RBBP8 (Fig. 1a, $P=0.0013$, Kruskal-Wallis test). Compared with the two copies of 1q21 PCM samples, RBBP8 was significantly increased in the 1q21 amplification samples ( $\geq 4$ copies) (Fig. 1a, $P=0.00055$, Wilcoxon test). The expression of RBBP8 in different molecular subtypes was different (Fig. $1 \mathrm{~b}, P<2.2 \mathrm{e}-16$, one-way analysis of variance analysis test). The expressions of RBBP8 in $\mathrm{CD} 1$ and PR subtypes were increasing, compared with the mean of all subtypes (Fig. $1 \mathrm{~b}, \mathrm{CD} 1, P \leq 0.05$; PR, $P \leq$ 0.0001 , unpaired $t$ test, two sided). Although MMSET subtype showed the lower RBBP8 expressions (Fig. 1b, $P<=0.0001$, unpaired $t$ test, two sided), and CD2, HY, $\mathrm{LB}$, and MAF subtypes were no significantly difference (Fig. 1b, $P>0.05$, unpaired $t$ test, two sided). In another molecular subtype classification, the expression of RBBP8 was also different (Fig. S2, $P<2.2 \mathrm{e}-16$, one-way analysis of variance analysis test). Compared with the average of all subtypes, $\mathrm{CD} 1, \mathrm{CD} 2, \mathrm{MF}$, and PR subtypes were showing higher RBBP8 expressions in GSE19784 data set (Fig. S2, CD1, $P \leq 0.05$; CD2, $P \leq 0.01$; MF, $P \leq 0.01$; PR, $P \leq$ 0.0001 , unpaired $t$ test, two sided). Although CTA and MMSET subtypes were showing the lower RBBP8 expressions (Fig. S2, $P \leq 0.0001$ unpaired $t$ test, two sided), and HY, MS, and myeloid subtypes were no significantly difference (Fig. S2, $P>0.05$, unpaired $t$ test, two sided).

\section{Expression level of RBBP8 in different ISS stages of PCM}

To investigate the expression pattern of RBBP8 in PCM, we analyzed expression profile of PCM from data set GSE24080. The gene expression level of RBBP8 was significantly related to the ISS grade of PCM (Fig. S1A, $P=$ 0.0012, Kruskal-Wallis test). Compared with ISS grade I PCM, ISS grade II and grade III PCM showed higher expression of RBBP8 (Fig. S1A, $P=0.0019, P=0.0049$, Wilcoxon test). This result indicated that high expression of RBBP8 was a sign of severity of PCM. We also studied the expression levels of RBBP8 in different ISS grades among different serotypes of PCM. According to the serum immunoglobulin component, PCM can be divided into eight types. We listed three common serotypes FLC (free light chain), IgA and IgG serotypes. We founded that there was a 
significant difference in the expression of RBBP8 among different ISS grades of IgG serotypes (Fig. S1B, $P=6.6 \mathrm{e}-$ 05, Kruskal-Wallis test). With the increase of ISS grades, the expression of RBBP8 gene was upregulated (Fig. S1B, $P=0.019, P=2.8 \mathrm{e}-05, P=0.058$, Wilcoxon test). The expression of RBBP8 was no significant difference in the different ISS grades of the IgA and FLC serotypes (Fig. S1B, IgA, $P=0.17$; FLC, $P=0.16$, Kruskal-Wallis test).

\section{Expression levels of RBBP8 was associated with relapse in PCM}

To investigate the relationship between the RBBP8 expression and the recurrence rate in PCM patients. We analyzed 585 cases PCM patients in GSE83503 data set. This data showed that RBBP8 expression was higher in relapsed patients compared with PCM patients who do not

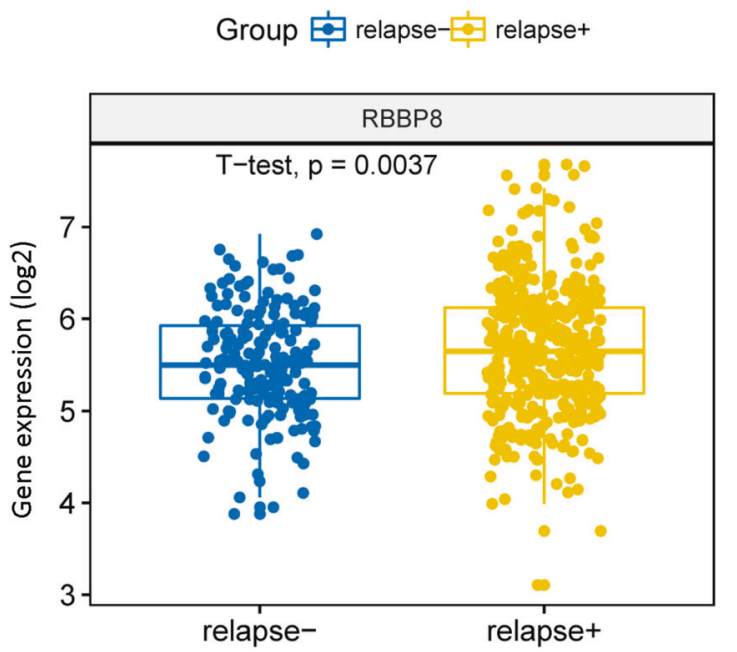

Fig. 2 The relationship between the expression of RBBP8 and recurrence, The $X$ axis represents no recurrence and recurrence, the $Y$ axis represents gene expression. RBBP8 gene expression was measured as $\log 2, P=0.0037$, unpaired $t$ test, two sided

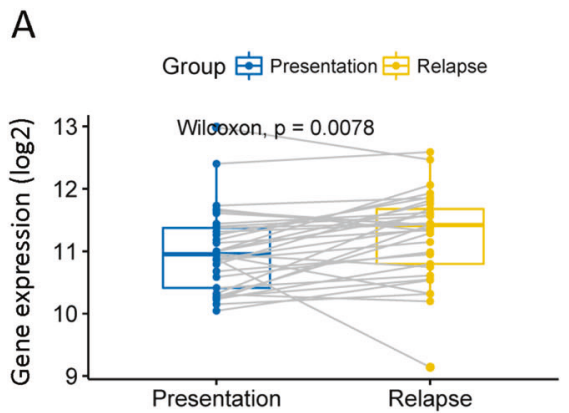

Fig. 3 Comparison of RBBP8 expression levels before and after recurrence in the same patient. a RBBP8 expression were significantly increase after relapse. The $X$ axis represents before and after recurrence and the $Y$ axis represents gene expression. RBBP8 gene expression was measured as $\log 2 . P=0.0078$, paired $t$ test, two sided. b RBBP8 relapse (Fig. 2, $P=0.0037$, Unpaired $t$ test, two sided). The data showed that the high initial expression level of RBBP8 determines the likelihood of recurrence.

To explored the relationship of RBBP8 expression between before and after recurrence in PCM. We asked 33 cases PCM patients with RBBP8 expression from data set GSE82307. RBBP8 expression significantly increases after relapse when compared with pre-relapse (Fig. 3a, $P=$ 0.0078 , paired $t$ test, two sided). We also described 19 cases PCM patients compared with the RBBP8 expression between baseline (diagnosis) and pre-1st (after induction chemotherapy) from the GSE19554 data set. There is an increasing trend between baseline and pre-1st (Fig. 3b, $P=$ 0.056 , paired $t$ test, two sided).

\section{Expression of RBBP8 genes predicted worse survival in PCM}

We studied the prognosis of PCM patients from the GSE24080 data set. The RBBP8 expression was highly correlated with the EFS and OS of PCM (Fig. 4, EFS, $P<$ 0.0001; OS, $P<0.0001$, log-rank test). The RBBP8-high group was associated with poor survival in PCM, whereas the RBBP8-low group had a good survival (Fig. 4). In addition, Cox regression analysis was performed to verify whether RBBP8 is an independent of clinical prognostic factor for PCM. In GSE24080 data set, it showed that RBBP8 ( $>11.08$ ), B2M $(\geq 3.5 \mathrm{mg} / \mathrm{l})$, MRI ( $\geq 3$ focal lesions) and BMPC ( $\geq 35 \%)$ were significantly associated with EFS (Table S1, $P=1.28 \mathrm{e}-03, P=3.54 \mathrm{e}-02, P=$ 2.40e-02, $P=1.74 \mathrm{e}-02$ ); and RBBP8, B2M, MRI were significantly associated with $\mathrm{OS}$ (Table $\mathrm{S} 1, P=1.31 \mathrm{e}-04$, $P=1.81 \mathrm{e}-02, P=9.72 \mathrm{e}-04)$. The hazard ratio of RBBP8 ( $>11.08$ vs $\leq 11.08$ ) in EFS was 1.57 (95\% CI, 1.19-2.06), and the hazard ratio in OS was 1.87 (95\% CI, 1.36-2.57). Between RBBP8-high group and RBBP8-low group, there was no significant in baseline patient characteristics such as

B

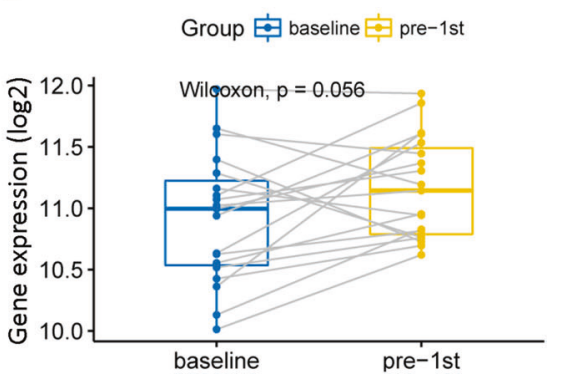

expression were increase between baseline (diagnosis) and pre-1st (after induction chemotherapy). The $X$ axis represents before and after treatment and the $Y$ axis represents gene expression $(\log 2), P=0.056$, paired $t$ test, two sided 
Fig. 4 RBBP8 was a prognostic factor in PCM. The $X$ axis represents time (month) and the $Y$ axis represents survival probability. Kaplan-Meier survival curves show that RBBP8 high expression predict poor event-free survival time (EFS) in PCM (Left plot), $P<$ 0.0001, log-rank test. KaplanMeier survival curves show that RBBP8 high expression predict poor overall survival (OS) in PCM (Right plot), $P<0.0001$, log-rank test
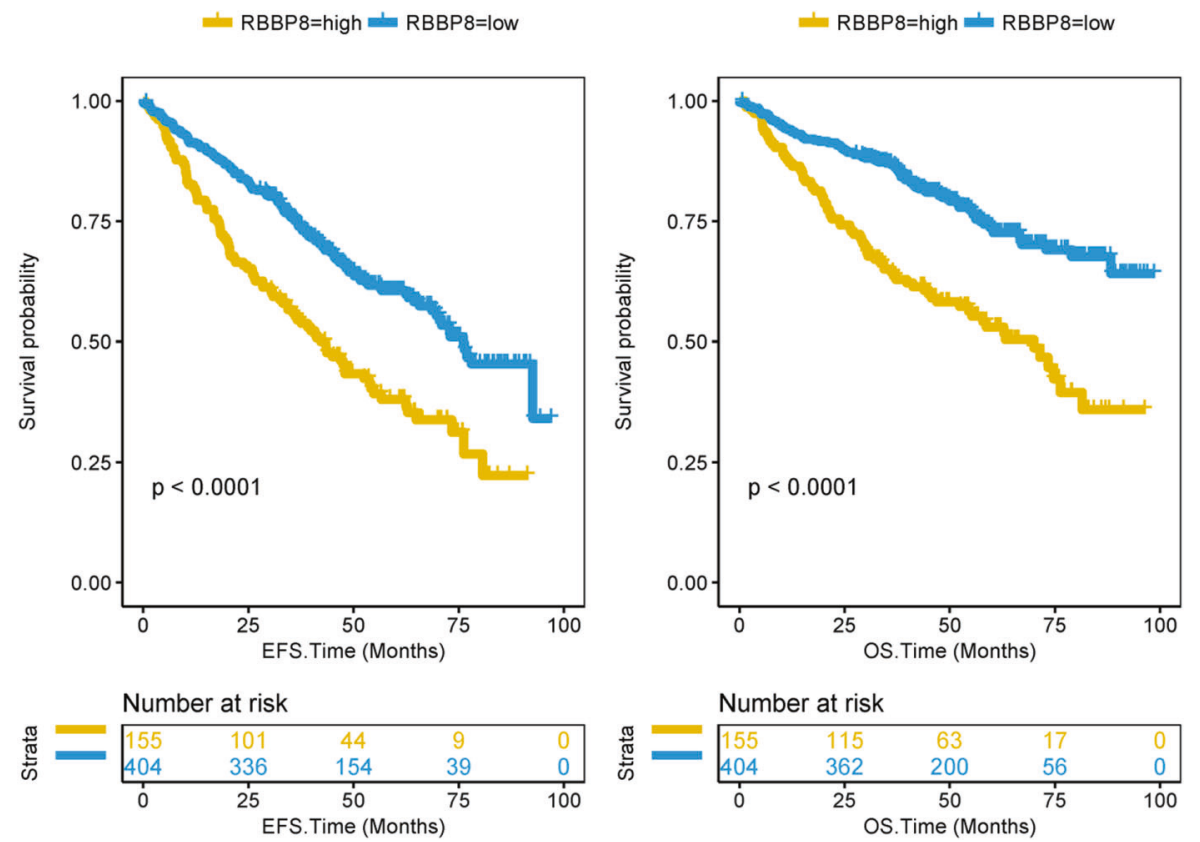

age, sex, race, and isotype (Table S2, $P>0.05$, Fisher exact test). Most of clinical characteristic were significantly different especially CRP, LDH, and MRI between these two groups (Table S2, $P<0.001$, unpaired $t$ test, two sided).

\section{The relationship between RBBP8 and cell cycle in PCM}

To investigate the biological role of RBBP8 expression in PCM, we selected the genes most relevant to RBBP8 for analysis. We found that RBBP8 was positively related to most cell cycle related genes and negatively related to a few cell cycles related genes. Among the 186 genes, 142 genes were upregulated with RBBP8 expression, and 44 genes were downregulated with RBBP8 expression. The heat map showed that top 12 upregulated genes and top 12 downregulated genes correlated with RBBP8 (Fig. 5a, $P<0.05$ ). To further elucidate the biologic role of RBBP8 in PCM, we performed GO analysis and showed the top 15 genes. The most significantly enriched pathway for all different expressed genes is DNA replication, cell division, and especially mitotic nuclear division (Fig. 5b, $P<0.05$ ). In DNA replication pathway, we showed that 15 different expressed genes (most of these genes are involved in cell division) are upregulated (Fig. 6, $P<0.0001$, unpaired $t$ test, two sided).

\section{Expression of RBBP8 in therapeutic response of PCM patients}

We analyzed RBBP8 expression and clinical treatment response in 238 PCM patients from data set GSE9782. The expression levels of RBBP8 was no significant difference in each treatment response group of biortezomib or Dex (Fig S3, Bortezomib, $P=0.37$; Dex, $P=0.53$, ANOVA test). We analyzed RBBP8 expression and clinical response of another 136 PCM patients from data set GSE39754. Similarly, there was no significant difference in the RBBP8 expression among all groups (Fig. S4, $P=0.26$, ANOVA test).

\section{Discussion}

Mutations in the RBBP8 gene cause tumors such as colorectal cancer and endometrial cancer [29, 30]. Low RBBP8 expression in many types of tumors (such as bladder cancer, ovarian cancer, and breast cancer) has a worse prognosis [21-23]. Presumably, the gene itself may be a tumor suppressor. However, little is known about the prognostic and biological significance of RBBP8 in PCM. We described the expression of RBBP8 in the gene expression profile of 1930 PCM samples (1878 PCM patients) from seven independent datasets, showing that the high RBBP8 expression predicts poorer survival level and relapse and affects the cell cycle in PCM.

PCM is a malignant tumor of terminally differentiated plasma cells. The survival time of PCM patients ranges from a few weeks to $>10$ years. As PCM is incurable, the significant differences in the survival of PCM, accurate stratification of patients with prognosis is essential to improve patient outcomes [31, 32]. Patients of PCM who predict of Monoclonal Gammopathy of Undetermined significance (MGUS) had better survival time [33]. 
Fig. 5 RBBP8 was closely related to cell cycle in PCM. a Heat map showed that different expression genes between the RBBP8-high group (red) and RBBP8-low group (green). Top 12 upregulated genes (red) and top 12 downregulated genes (green) were showed. The two bar plots are foldchange $(\log 2$, left) and $P$ value $(-\log 10$, right), respectively. b GO analysis of the top 15 pathways showed that RBBP8 was mostly involved DNA replication, cell division, and mitotic nuclear division. $P$ value $(-\log 10)$

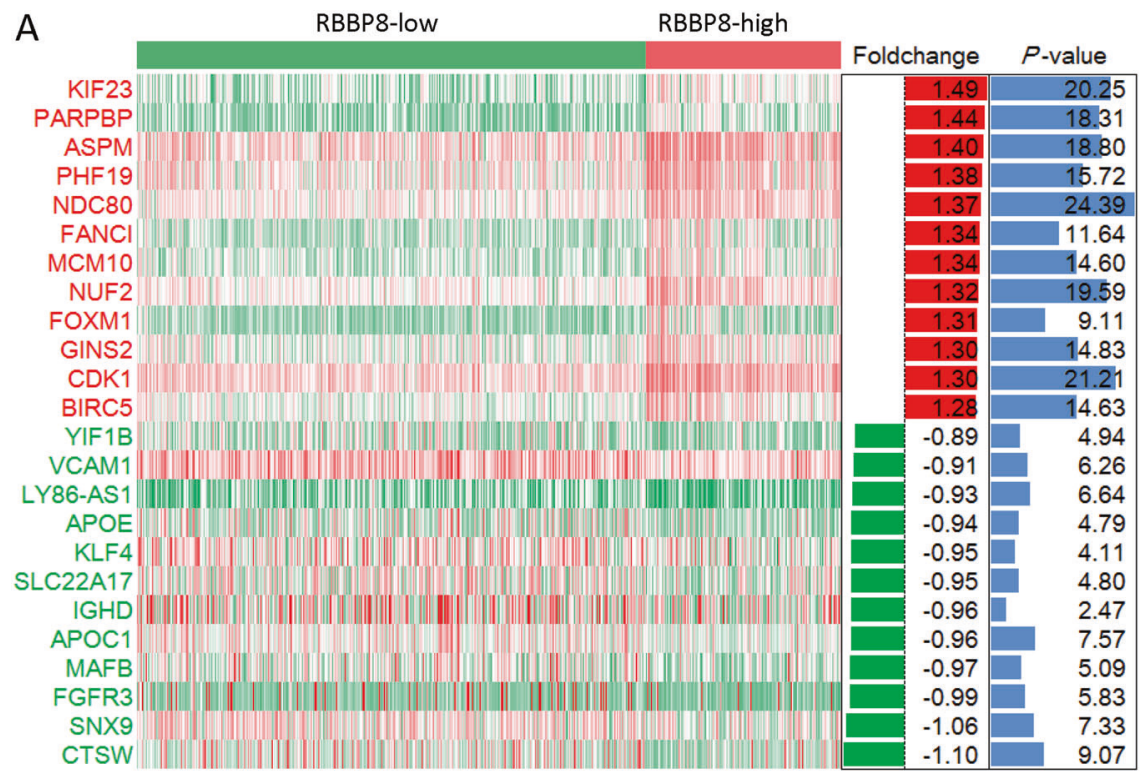

B

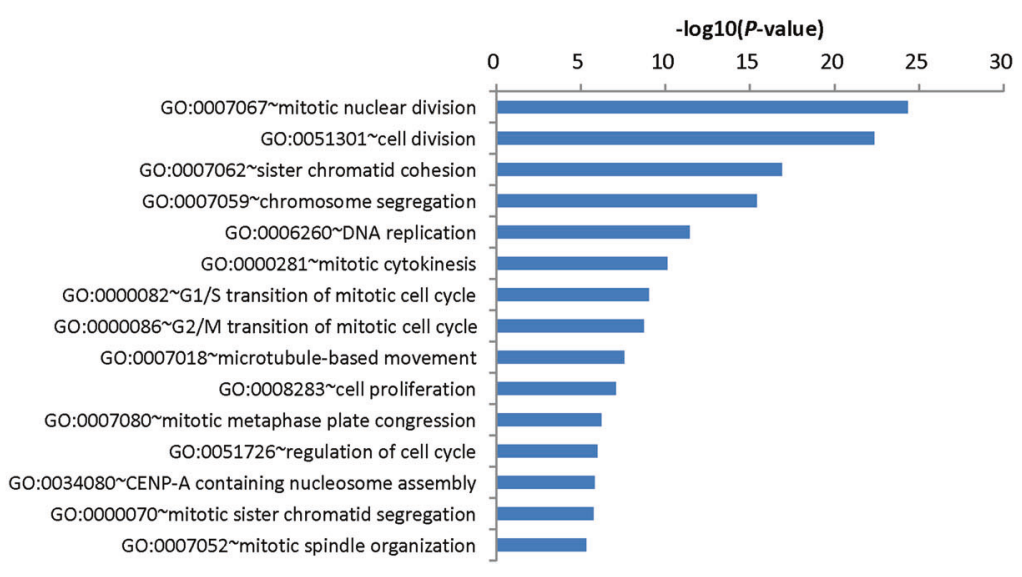

Whole-body magnetic resonance imaging was used as a risk stratification tool for asymptomatic PCM [34]. In our analysis, RBBP8 expression levels were significantly associated with EFS and OS in PCM. High expression of RBBP8 predicts worse prognosis. Like B2M and MRI, RBBP8 can be considered as independent prognostic factor for PCM. In different serotypes or molecular typing in PCM, RBBP8 expression was different. This indicates that detecting RBBP8 expression levels in the PCM patients can be predicted the ISS stage in certain serotypes and can predict the prognosis in certain molecular typing.

RBBP8 is a protein involved in transcription, DNA replication, DNA repair, and a key checkpoint of $\mathrm{G}_{1}$-phase and $\mathrm{G}_{2}$-phase in cell cycle. We found that RBBP8 played an important role in the cell cycle. In particular, we found that many genes involved in the cell cycle are upregulated in the DNA replication pathway. Component of the BRCA1RBBP8 complex regulates CHEK1 activation and controls the cell cycle G2/M checkpoint for DNA damage [35-37]. RBBP8 identified as an candidate oncogene participated in regulating cell cycle [38]. This function of RBBP8 was also observed in other tumors $[16,19,39]$. DNA replication and cell proliferation may be the cause of progression in PCM patients. As revealed above, RBBP8 played a significant role in the cell cycle in PCM. Therefore, the RBBP8 gene may predict poor PCM survival levels by regulating DNA replication pathways. In the future, relevant genes affecting these pathways should be in-depth studied.

PCM is a disease with a high recurrence rate. Researcher has clarified the potential causes of PCM relapse in the previous [40]. Here, we found the relationship between RBBP8 gene expression and recurrence. This finding indicated that the RBBP8 expression is positively correlated with relapse in PCM. It showed that the high initial expression level of the RBBP8 gene determines the likelihood of recurrence. These results suggested that high expression of RBBP8 can predict more likely to relapse in PCM patients.

However, further research is needed to study the molecular mechanisms of RBBP8 in the development of PCM. 

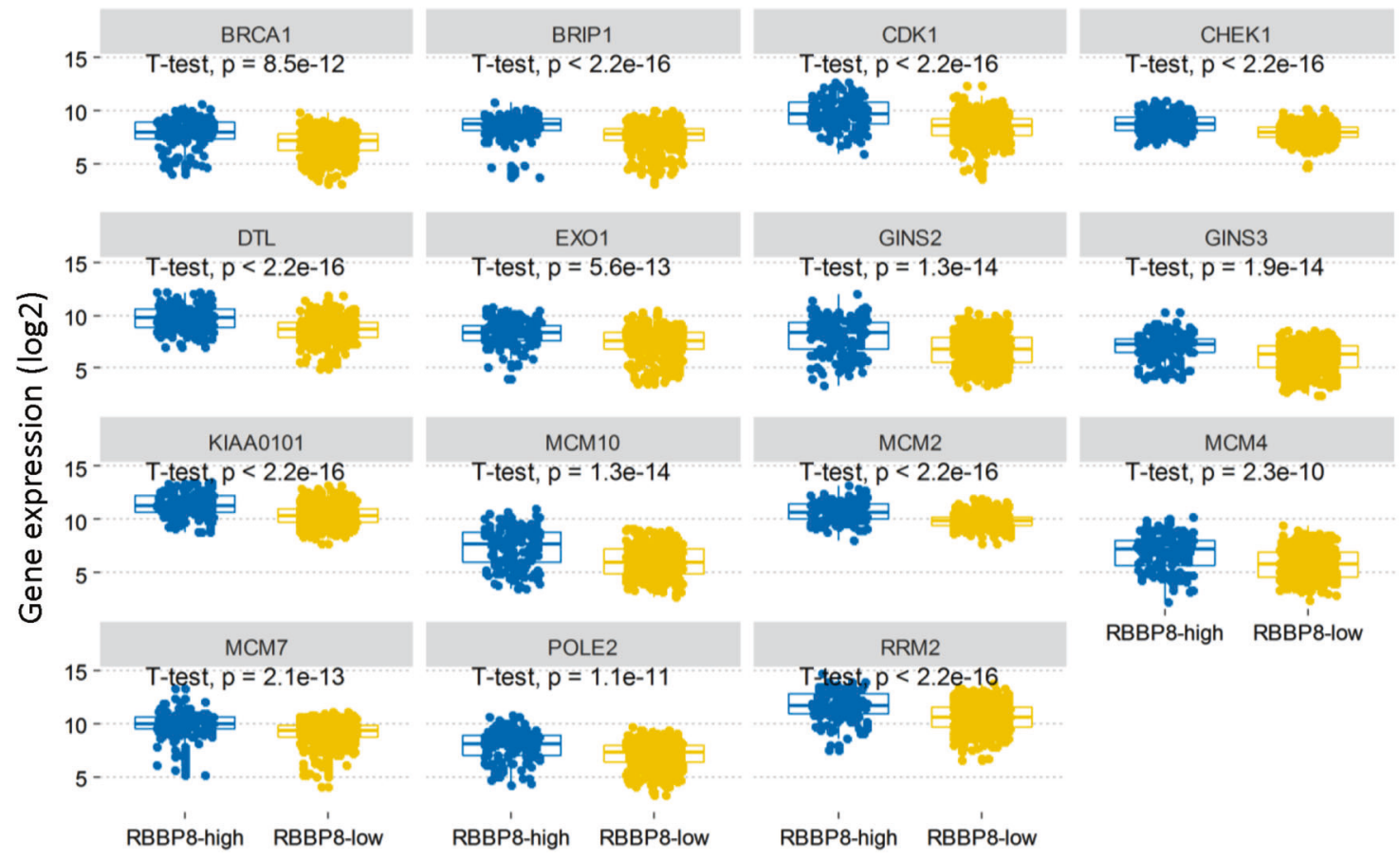

Fig. 6 Differentially expressed genes in the DNA replication pathway were displayed. All genes are upregulated. BRCA1, $P=8.5 \mathrm{e}-12$; BRIP, CDK1, CHEK1, DHL, KIAA0101, MCM2, RRM2, $P<2.2 \mathrm{e}-16$;

GINS2, MCM10, $P=1.3 \mathrm{e}-14$, EXO1, $P=5.6 \mathrm{e}-13$, G1NS3, $P=1.9 \mathrm{e}-$ 14, MCM4, $P=2.3 \mathrm{e}-10$, MCM7, $P=2.1 \mathrm{e}-13$, POLE2, $P=1.1 \mathrm{e}-11$, unpaired $t$ test, two sided

Such as, conduct some experimental studies to further verify the results. This time we only described a single gene expression biomarker, and future studies can be combined with multiple gene expression biomarkers to assess the prognosis of PCM.

In summary, high expression of RBBP8 gene predicts worse prognosis in PCM patients. RBBP8 may play an important role in the cell cycle, especially in DNA replication in PCM. High expression of RBBP8 predicts higher relapse rate of PCM. RBBP8 can be considered as a new biomarker for PCM prognosis.

Acknowledgements This work was funded by interdisciplinary medicine Seed Fund of Peking University (BMU2018MB004), Beijing Natural Science Foundation (7132183), China Health Promotion Foundation (CHPF-zlkysx-001).

Author contributions HMJ, XLY, XRZ, and WYL conceived the project. WLZ, YS, and XH analyzed the data. WLZ, YS, XH, XNL, YZ, ZZY, PY, JW, KH, XRZ, and HMJ contributed towards the interpretation of the data. All authors wrote and approved the final manuscript.

\section{Compliance with ethical standards}

Conflict of interest The authors declare that they have no conflict of interest.

Publisher's note: Springer Nature remains neutral with regard to jurisdictional claims in published maps and institutional affiliations.
Open Access This article is licensed under a Creative Commons Attribution 4.0 International License, which permits use, sharing, adaptation, distribution and reproduction in any medium or format, as long as you give appropriate credit to the original author(s) and the source, provide a link to the Creative Commons license, and indicate if changes were made. The images or other third party material in this article are included in the article's Creative Commons license, unless indicated otherwise in a credit line to the material. If material is not included in the article's Creative Commons license and your intended use is not permitted by statutory regulation or exceeds the permitted use, you will need to obtain permission directly from the copyright holder. To view a copy of this license, visit http://creativecommons. org/licenses/by/4.0/.

\section{References}

1. Kyle RA, Rajkumar SV. Multiple myeloma. N Engl J Med. 2004;351:1860-73.

2. Costa LJ, Gonsalves WI, Kumar SK. Early mortality in multiple myeloma. Leukemia. 2015;29:1616-8.

3. Kumar SK, Rajkumar SV, Dispenzieri A, Lacy MQ, Hayman SR, Buadi FK, et al. Improved survival in multiple myeloma and the impact of novel therapies. Blood. 2008;111:2516-20.

4. Pineda-Roman M, Zangari M, Haessler J, Anaissie E, Tricot G, van Rhee F, et al. Sustained complete remissions in multiple myeloma linked to bortezomib in total therapy 3: comparison with total therapy 2. Br J Haematol. 2008;140:625-34.

5. Barlogie B, Tricot G, Anaissie E, Shaughnessy J, Rasmussen E, van Rhee F, et al. Thalidomide and hematopoietic-cell transplantation for multiple myeloma. N Engl J Med. 2006;354:1021-30.

6. McCarthy PL, Owzar K, Hofmeister CC, Hurd DD, Hassoun H, Richardson PG, et al. Lenalidomide after stem-cell transplantation for multiple myeloma. N Engl J Med. 2012;366:1770-81. 
7. Abdelkefi A, Ladeb S, Torjman L, Othman TB, Lakhal A, Romdhane NB, et al. Single autologous stem-cell transplantation followed by maintenance therapy with thalidomide is super ior to double autologous transplantation in multiple myeloma: results of a multicenter randomized cl inical trial. Blood. 2008;111:1805-10.

8. Bergsagel PL, Kuehl WM, Zhan F, Sawyer J, Barlogie B, Shaughnessy $\mathrm{J} \mathrm{Jr}$, et al. Cyclin D dysregulation: an early and unifying pathogenic event in multiple myeloma. Blood. 2005;106:296-303.

9. Zhan F, Huang Y, Colla S, Stewart JP, Hanamura I, Gupta S, et al. The molecular classification of multiple myeloma. Blood. 2006;108:2020-8.

10. Broyl A, Hose D, Lokhorst H, de Knegt Y, Peeters J, Jauch A, et al. Gene expression profiling for molecular classification of multiple myeloma in newly diagnosed patients. Blood. 2010;116:2543-53.

11. Mulligan G, Mitsiades C, Bryant B, Zhan F, Chng WJ, Roels S, et al. Gene expression profiling and correlation with outcome in clinical trials of the proteasome inhibitor bortezomib. Blood. 2007;109:3177-88.

12. Chauhan D, Tian Z, Nicholson B, Kumar KG, Zhou B, Carrasco $\mathrm{R}$, et al. A small molecule inhibitor of ubiquitin-specific protease7 induces apoptosis in multiple myeloma cells and overcomes bortezomib resistance. Cancer Cell. 2012;22:345-58.

13. Liu F, Lee WH. CtIP activates its own and cyclin D1 promoters via the E2F/RB pathway during G1/S progression. Mol Cell Biol. 2006;26:3124-34.

14. Ahmed KM, Tsai CY, Lee WH. Derepression of HMGA2 via removal of ZBRK1/BRCA1/CtIP complex enhances mammary tumorigenesis. J Biol Chem. 2010;285:4464-71.

15. Chen PL, Liu F, Cai S, Lin X, Li A, Chen Y, et al. Inactivation of CtIP leads to early embryonic lethality mediated by G1 restraint and to tumorigenesis by haploid insufficiency. Mol Cell Biol. 2005;25:3535-42.

16. Yun MH, Hiom K. CtIP-BRCA1 modulates the choice of DNA double-strand-break repair pathway throughout the cell cycle. Nature. 2009;459:460-3.

17. Davies OR, Forment JV, Sun M, Belotserkovskaya R, Coates J, Galanty Y, et al. CtIP tetramer assembly is required for DNA-end resection and repair. Nat Struct Mol Biol. 2015;22:150-7.

18. Gu B, Chen PL. Expression of PCNA-binding domain of CtIP, a motif required for CtIP localization at DNA replication foci, causes DNA damage and activation of DNA damage checkpoint. Cell Cycle. 2009;8:1409-20.

19. Kremer PH, Koeleman BP, Pawlikowska L, Weinsheimer S, Bendjilali N, Sidney S, et al. Evaluation of genetic risk loci for intracranial aneurysms in sporadic arteriovenous malformations of the brain. J Neurol Neurosurg Psychiatry. 2015;86:524-9.

20. Mijnes J, Veeck J, Gaisa NT, Burghardt E, de Ruijter TC, Gostek $\mathrm{S}$, et al. Promoter methylation of DNA damage repair (DDR) genes in human tumor entities: RBBP8/CtIP is almost exclusively methylated in bladder cancer. Clin Epigenetics. 2018;10:15.

21. Quaye L, Dafou D, Ramus SJ, Song H, Gentry-Maharaj A, Maharaj AG, et al. Functional complementation studies identify candidate genes and common genetic variants associated wi th ovarian cancer survival. Hum Mol Genet. 2009;18:1869-78.

22. Soria-Bretones I, Saez C, Ruiz-Borrego M, Japon MA, Huertas P. Prognostic value of CtIP/RBBP8 expression in breast cancer. Cancer Med. 2013;2:774-83.

23. Wang J, Ding Q, Fujimori H, Motegi A, Miki Y, Masutani M. Loss of CtIP disturbs homologous recombination repair and sensitizes breast cancer cells to PARP inhi bitors. Oncotarget. 2016;7:7701-14

24. Xu J, Lv S, Qin Y, Shu F, Xu Y, Chen J, et al. TRB3 interacts with CtIP and is overexpressed in certain cancers. Biochim Biophys Acta. 2007;1770:273-8.

25. Shi L, Campbell G, Jones WD, Campagne F, Wen Z, Walker SJ, et al. The MicroArray Quality Control (MAQC)-II study of common practices for the development and validation of microarray-based predictive models. Nat Biotechnol. 2010;28:827-38.

26. Miannay B, Minvielle S, Roux O, Drouin P, Avet-Loiseau H. Gu“'irinCharbonnel $\mathrm{C}$, et al. Logic programming reveals alteration of key transcription factors in multiple myeloma. Sci Rep. 2017;7:9257.

27. Weinhold N, Ashby C, Rasche L, Chavan SS, Stein C, Stephens OW, et al. Clonal selection and double-hit events involving tumor suppressor genes underlie relapse in myeloma. Blood. 2016;128:1735-44.

28. Zhou W, Yang Y, Xia J, Wang H, Salama ME, Xiong W, et al. NEK2 induces drug resistance mainly through activation of efflux drug pumps and is associated with po or prognosis in myeloma and other cancers. Cancer Cell. 2013;23:48-62.

29. Vilkki S, Launonen V, Karhu A, Sistonen P, Vastrik I, Aaltonen LA. Screening for microsatellite instability target genes in colorectal cancers. J Med Genet. 2002;39:785-9.

30. Bilbao C, Ramírez R, Rodríguez G, Falcón O, León L, DíazChico N, et al. Double strand break repair components are frequent targets of microsatellite instability in endometri al cancer. Eur J Cancer. 2010;46:2821-7.

31. Rajkumar SV, Dimopoulos MA, Palumbo A, Blade J, Merlini G, Mateos MV, et al. International Myeloma Working Group updated criteria for the diagnosis of multiple myeloma. Lancet Oncol. 2014;15:e538-48.

32. Chng WJ, Dispenzieri A, Chim CS, Fonseca R, Goldschmidt H, Lentzsch S, et al. IMWG consensus on risk stratification in multiple myeloma. Leukemia. 2014;28:269-77.

33. Sigurdardottir EE, Turesson I, Lund SH, Lindqvist EK, Mailankody $\mathrm{S}$, Korde $\mathrm{N}$, et al. The role of diagnosis and clinical follow-up of monoclonal gammopathy of undetermined significance on survival in multiple myeloma. JAMA Oncol. 2015;1:168-74.

34. Hillengass J, Fechtner K, Weber MA, Bäuerle T, Ayyaz S, Heiss $\mathrm{C}$, et al. Prognostic significance of focal lesions in whole-body magnetic resonance imaging in patients with as ymptomatic multiple myeloma. J Clin Oncol. 2010;28:1606-10.

35. Yu X, Fu S, Lai M, Baer R, Chen J. BRCA1 ubiquitinates its phosphorylation-dependent binding partner CtIP. Genes Dev. 2006;20:1721-6.

36. Sartori AA, Lukas C, Coates J, Mistrik M, Fu S, Bartek J, et al. Human CtIP promotes DNA end resection. Nature. 2007;450:509-14.

37. Steger M, Murina O, Huhn D, Ferretti LP, Walser R, Hanggi K, et al. Prolyl isomerase PIN1 regulates DNA double-strand break repair by counteracting DNA end resection. Mol Cell. 2013;50:333-43.

38. Ciriello G, Cerami E, Sander C, Schultz N. Mutual exclusivity analysis identifies oncogenic network modules. Genome Res. 2012;22:398-406.

39. Bothmer A, Rommel PC, Gazumyan A, Polato F, Reczek CR, Muellenbeck MF, et al. Mechanism of DNA resection during intrachromosomal recombination and immunoglobulin class switching. J Exp Med. 2013;210:115-23.

40. Chan Chung KC, Tiedemann RE. Getting to the root of the problem: the causes of relapse in multiple myeloma. Expert Rev Anticancer Ther. 2014;14:251-4. 